\title{
Textural Features and Relevance Feedback for Image Retrieval
}

\author{
E. Di Sciascio, G. Piscitelli, A. Celentano* \\ Dip. Elettrotecnica ed Elettronica, Politecnico di Bari, Via E. \\ Orabona 4, I-70125, Bari Italy \\ * Dip. Matematica Applicata e Informatica, Università Ca' \\ Foscari di Venezia, Via Torino 155, I-30173 Mestre (VE), Italia
}

\begin{abstract}
This paper focuses on the retrieval of complex images based on their texture content. We use GMRF for texture discrimination and a region-growing algorithm for texture segmentation. Relevance feedback is introduced to improve retrieval accuracy.
\end{abstract}

\section{Keywords}

Textures, relevance feedback, image features, Gaussian Markovian Random Fields.

\section{INTRODUCTION}

Content-based image classification, indexing and retrieval requires semantic interpretation and cannot be afforded with current technology. A surrogate of semantic interpretation is the computation of visual features that can be used as quantitative parameters for the identification of similar images. Thus, the problem of retrieving images with some content is substituted with the problem of retrieving images visually close to a target one. Image features like colors, contours, textures, have been used in various systems. Related work includes, but it is not limited to Flickner (1995), Bach (1996), Ma (1997), Popat (1997), Rui (1997), Celentano (1998). Texture is one of the characteristics the human beings normally use to perceive the content of an image. In this paper we focus on the retrieval of complex images based on their textural content.

We evaluated various texture analysis algorithms, i.e. Spatial Grey-Level Cooccurrence Matrices (SGLCM) (Raalick 1973, Davis, 1989), Power Spectral Density (PSD) (Dyer, 1990), and Gaussian Markovian Random Fields (GMRF) (Khotanzad, 1987). We selected GMRF for texture discrimination; further we designed a simple region growing textures segmentation algorithm. Relevance 
feedback, a technique widely used in conventional text retrieval, was introduced to improve retrieval accuracy.

\section{TEXTURE ANALYSIS}

Image retrieval based on texture analysis requires image segmentation during database population in order to identify areas having homogeneous texture content. Segmentation is based on measures obtained by the application of the GMRF algorithm. Following the method proposed by Reed (1990) we subdivide the image in measurement windows. A sub-sampling is performed on each image, associating a measurement window to each $n$ pixels (in our implementation a $32 \times 32$ pixels window for each 16 pixels). The image is hence associated to a sub-sampled "textural" image where each texture element (texel) is associated with a vector of coefficients $f$, relative to the corresponding measurement window, that characterises it. For a $\mathrm{N} \times \mathrm{M}$ image the size of the corresponding textured image is:

$$
K \times L=\left[2 \cdot \text { floor }\left(\frac{N}{32}\right)-1\right] \times\left[2 \cdot \text { floor }\left(\frac{M}{32}\right)-1\right] \text {. }
$$

Similarity measurement between texels requires the evaluation of the distance between their corresponding vector coefficients. We used a simple metric that consists of the sum of the differences of corresponding coefficients, normalised with their arithmetical mean; i.e., given two texels $\mathrm{Ti}$ and $\mathrm{Tj}$ and the corresponding vectors $f i$ and $f j$, the distance is:

$$
d\left(T_{i}, T_{j}\right)=\sum_{r=1}^{R} 2 \times\left|\frac{f_{i}(r)-f_{j}(r)}{f_{i}(r)+f_{j}(r)}\right|
$$

where $R$ is the number of coefficients in the vector ( $R=7$ for GMRF). This metric allows us to measure differences between coefficients regardless of their absolute values, and the distance between two texels does not depend on their ordering. The determination of starting seeds is crucial; for each texel $T i$ the texel having the smaller distance in a $\mathrm{NN}=3 \times 3$ is computed:

$$
T_{\min } \ni^{\prime} d\left(T_{\min }, T_{i}\right)=\min _{T_{j} \in N N}\left\{d\left(T_{i}, T_{j}\right)\right\}
$$

The texel $T i$ can be assumed as starting seed if all other texels within the neighbourhood have a distance which is less than a given threshold $t$ :

$T i=$ seed $\Leftrightarrow \forall T_{j} \in N N: d\left(T_{i}, T_{j}\right) \leq d\left(T_{i}, T_{\min }\right) \cdot(1+t)$

The segmentation process, starting from identified seed texels is performed in a way that can be assimilated to the diffusion of a particle towards texels having a minimum distance from the starting seed. The diffusion is forbidden if the distance is higher than the threshold or if all texels in the neighbourhood of the seed have already been included. Detected texture areas are characterised by two vectors, one 
storing mean values and the other storing variance values for each of the rectangular windows the area is subdivided into.

\section{QUERY PROCESSING}

Queries are submitted by example either as a sample texture image or selecting a rectangular area within a submitted image; the selected area is subdivided into windows according to segmentation stage. Then, for each window the coefficients are extracted by applying the GMRF model and the correspondent feature vectors are created. The similarity measure between the selected query area, represented by its feature vector $\mathbf{Q}$, and textured areas of images in the database, represented by their feature vectors $\mathbf{T} i$ is performed computing the Euclidean distance between vectors storing associated mean values. Such distance is normalised with associated variance values providing the following expression:

$$
d(\boldsymbol{Q}, \boldsymbol{T i})=\sqrt{\sum_{j=1}^{4} \frac{\left(f_{j}(Q)-f_{j}(T i)\right)^{2}}{\sigma_{j}^{2}(T i)}}
$$

Resulting retrieved image are indexed according to increasing distance score.

Relevance feedback is a query refinement technique widely used in text retrieval system (Salton, 1989). Due to uncertainty in the interpretation of word meaning, some of the retrieved documents can be not relevant to the user, while other lowranked documents can be more meaningful. A modified query that increases the contribution of the terms of meaningful documents, and lowers the contribution of terms of less relevant documents, can achieve a better retrieval performance. Uncertainty is even more present in image retrieval, due to the weaker correspondence between the features and the image as perceived by the user, i.e., the system may not match the user perception of similarity. Relevance feedback, consisting in marking some of the retrieved images as good or bad, can improve the computation of similarity by giving different weights to the compared features (Celentano, 1998, Rui, 1997).

In Salton (1989), it is proposed to obtain the modified query Q' by adding to the original query vector $\mathbf{Q}$ the vectors of relevant documents $\mathbf{X i}$ and subtracting the irrelevant ones $\mathrm{Yi}$, both weighted with suitable coefficients:

$$
Q^{\prime}=Q+\chi \sum_{i=1}^{N_{r e l}} X_{i}-\delta \sum_{i=1}^{M_{i r e l}} Y_{i}
$$

This basic expression has been modified here in order to adapt it to our particular domain of operation. We compute the nearing operation of the new query to the relevant texture set, while preserving the different validity domain of the features in the query vector, by adopting a simple arithmetical mean between the central vector of the relevant queries $\mathrm{N}_{\text {rel }}$ and the original query $\boldsymbol{Q}$ : 


$$
Q^{\prime}=\frac{Q+\frac{1}{N_{r e l}} \sum_{i=1}^{N_{r l}} X_{i}}{2}
$$

that corresponds to expression (6) with $\chi=\frac{1}{2 \cdot N_{\text {rel }}}, \delta=0$.

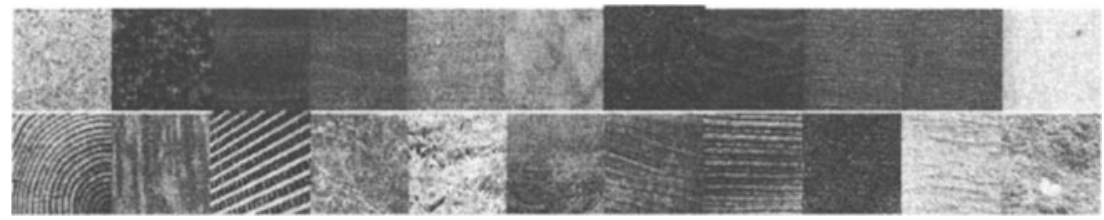

\section{Figure 1 Test textures}

It is worth noting that, though the application of relevance feedback in our framework has proved to be effective, a conceptual difference exists with respect to the same approach applied to text documents. In that case, an index term either appears in a document, with some weight, or it does not appear. In image retrieval, basically all index terms are present in the whole collection, though with different contributions. The relevance feedback operates by changing the amount of contribution of the feature components.
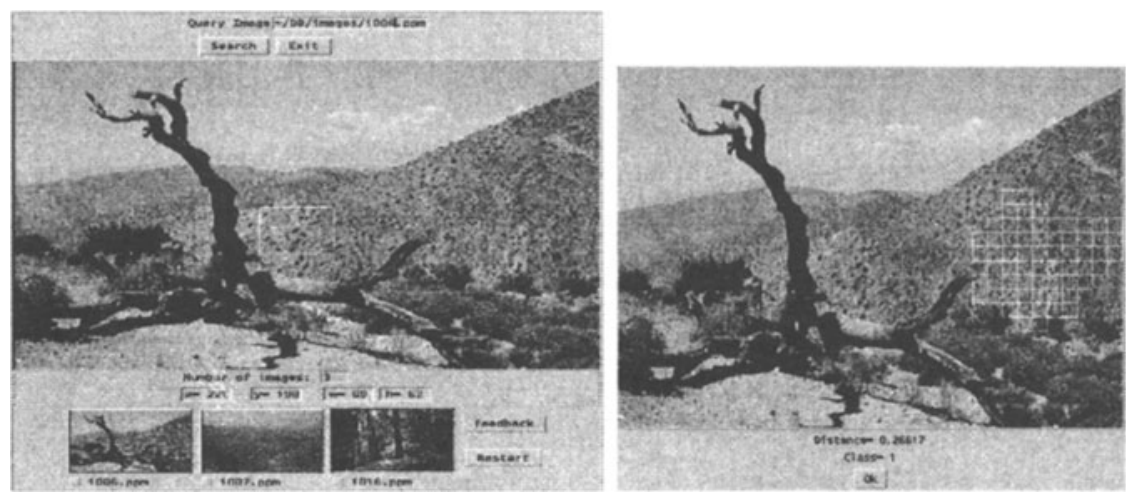

Figure 2 An example retrieval: query and Figure 3 Relevant texture area for the the three highest ranking retrieved images. retrieved image in figure 2.

\section{EXPERIMENTAL RESULTS AND CONCLUSIONS}

The system has been implemented in Java and Tcl-Tk under the Linux operating system. The evaluation of the texture discrimination capabilities has been carried out for three algorithms, namely SGLCM, PSD and GMRF. A classification process has been implemented starting from 22 texture images, 512 by 512 pixels, shown in figure 1 . From each image a sub-image 256 by 256 (parent texture) has been extracted to serve as a reference and six others 128 by 128 (children textures) 
have been extracted to serve as queries. The classification process has been considered positive if the children textures were correctly associated to the parent one. Obtained results, in terms of hit-rate and retrieval time (on a PC equipped with a Pentium $100 \mathrm{CPU}$ and $16 \mathrm{Mbyte}$ RAM) are : $88.64 \%$ and $17.24 \mathrm{~s}$ for SGLCM, 93.94\% and $14.78 \mathrm{~s}$ for GMRF, and $87.12 \%$ and $58.95 \mathrm{~s}$ for PSD. We experimented the segmentation algorithm starting from measures obtained applying SAR and CSAR models, which came out as the most effective in our test database characterisation. We assumed a measure window having $32 \times 32$ pixels size moving along the image with a 16 pixels step. We set the threshold to 0.2 , in order to obtain a high selectivity in the texture discrimination process. The experiments proved that the segmentation algorithm performed well enough, identifying homogeneous texture areas. It must also be admitted that the conservative threshold and the lack of a merge of similar textured areas may produce not completely satisfactory results.

The system has a low level of human interaction: the whole analysis stage, i.e. texture feature extraction and segmentation, is automatic, user intervention being requested only to provide relevance feedback. To perform a query, the user can either submit a sample texture image or select a rectangular area in a picture selected from the database. Retrieved images are ranked in decreasing order of similarity. The number of retrieved images, displayed according to the highest ranking can be adjusted by the user.

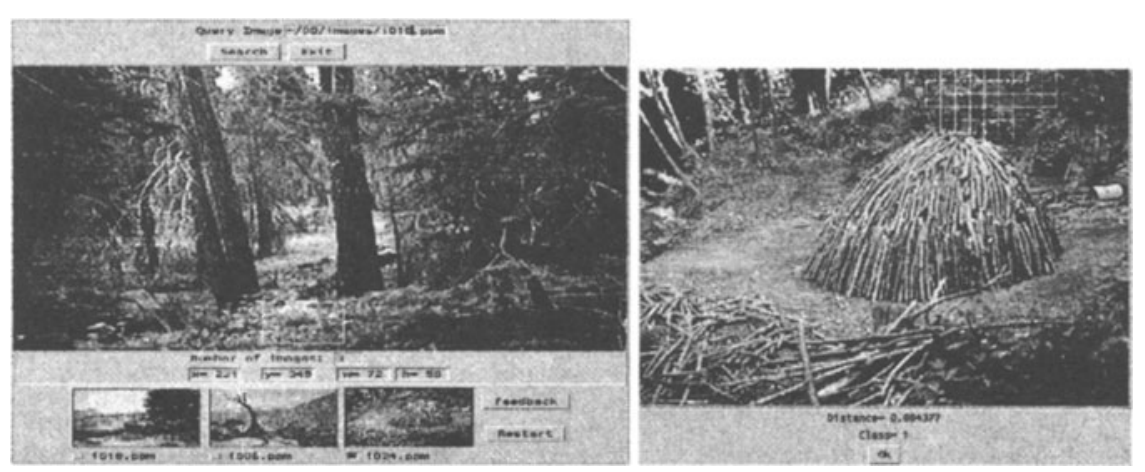

Figure 4 The image with check-button push- Figure 5 Relevant texture area for ed was identified as relevant by the user. the retrieved image in figure 4.

Figure 2 shows an example retrieval by pictorial example on the image collection: the query image is shown on the top left and the queried area is in the white bordered rectangle; retrieved images are displayed in the lower part, ranked in decreasing order of similarity. Figure 3 shows, for the highest ranking retrieved image, the area found as most similar to the query. Figure 4 shows another example of query and retrieval. Figure 5 shows the area found as most similar in image 24, i.e. the highest ranking one in the retrieved set. As it can be noticed, retrieval results may not appear satisfactory: the second higher ranking image does not appear to have the same textural content of the query and the original image was 
not retrieved, though the other two images that are present in the retrieved set are visually similar to the query. By selecting as relevant the image that visually appears more similar to the query (the one with check button marked in figure 4), relevance feedback was applied, stressing distinguished feature in the "relevant" image and obtaining the results displayed in figure 6 . It can be noticed how the selected image now ranks highest, the more different one has been withdrawn and the image the query area was selected from now appears in the retrieved set.

A classification experiment was undertaken considering a small repository of 40 images picturing natural scenes. Despite the toy size of the collection we believe the test allows to sufficiently evaluate the proposed approach. Each image was used as query, selecting a texture area, against the whole database; retrieval results were considered correct if the original image was retrieved in the first three higher ranking pictures. Results proved a good performance of the system: original images were correctly retrieved with a percentage of $77.5 \%$. This percentage raised to $100 \%$ after relevance feedback.

Further work is in progress in two main directions :improvement of the segmentation algorithm to allow automatic merging of similar texture areas, and integration of the system with other feature extraction approaches we already developed, including extension to coloured textures.

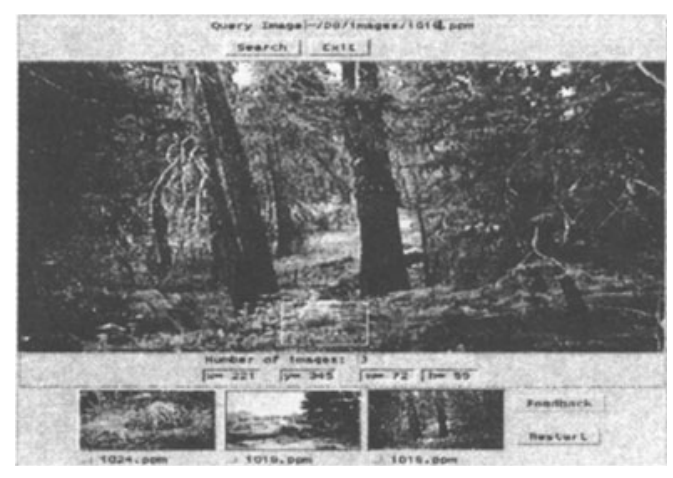

Figure 6 Query and retrieval results after application of relevance feedback.

\section{ACKNOWLEDGEMENTS}

This work was carried out in the framework of the projects Image Coding in Mobile Communication (CNR) and Basi di dati evolute: modelli, metodi e strumenti (MURST).

\section{REFERENCES}

Bach R. et al. (1996) The Virage Image Search Engine: An open framework for image management, SPIE, 2670, 76-87.

Celentano A., Di Sciascio E. (1998) Features Integration and Relevance Feedback 
Analysis in Image Similarity Evaluation, Journal of Electronic Imaging, 7,2, 1998. Davis L.S., Johns S.A., Aggrawal J.K. (1989) Texture Analysis Using Generalized Co-occurrence Matrices, IEEE Trans. on PAMI, 1, 2, 251-9.

Dyer C.R., Rosenfeld A. (1990) Fourier Texture Features: Suppression of Aperture Effects, IEEE Trans. on Syst., Man., Cybern., 6, 10, 703-5.

Flickner M. et al. (1995) Query by Image and Video Content: The QBIC System, IEEE Computer, 28, 9, 23-31.

Khotanzad A., Kashyap R.L.(1987) Features Selection for Texture Recognition Based on Image Synthesis, IEEE Trans. on Syst., Man., Cybern., 17, 11, 1087-95.

Ma W.Y., Manjunath B.S. (1997) NETRA: A toolbox for navigating large image databases, in Proc. of IEEE ICIP-97.

Popat C., Picard R.W. (1997) Cluster-based Probability Model and its Application to Image and Texture Processing, IEEE Trans. on Image. Processing, 6, 2, 268-84.

Raalick, R.M., Shanmugam K, Disnstein I. (1973) Textural features for Image Classification, IEEE Trans. On Syst., Man, Cybern., 3, 11, 610-21.

Reed T.R., Wechsler H., Werman M. (1990) Texture Segmentation Using a Diffusion Region Growing Technique, Pattern Recognition, 23, 9, 953-60.

Rui Y, Huang T.S., Mehrotra S. (1997) Content-based Image Retrieval with Relevance Feedback in MARS, in proc. IEEE ICIP-97.

Salton, G. (1989) Automatic Text Processing, Addison Wesley

\section{BIOGRAPHIES}

Eugenio Di Sciascio received the laurea "cum laude" degree in Electronic Engineering from University of Bari in 1989 and the Ph.D. degree in 1994 from Technical University of Bari. In 1992 he joined the University of Lecce as an assistant professor. Since 1995 he holds a permanent position at Technical University of Bari where he currently teaches "Fundamentals of Computer Science" and "Information Systems". His research interests include image processing and multimedia information systems. He has published over 35 papers on international journals and conference proceedings $\mathrm{He}$ is member of IEEE and ACM.

Giacomo Piscitelli received the degree in physics with honours from the University of Bari in 1966. Currently he is professor of Operating Systems at the Polytechnic of Bari. His research interests are in the areas of Operating Systems, Flexible Manufacturing Systems Management, Multimedia Information Systems.

Augusto Celentano received the degree in Electronic Engineering at Techincal University of Milan in 1975. He is now Full Professor of Computer Science at University "Ca' Foscari" of Venice. His research interests include multimedia information systems, collaborative systems, and distance education. He has published over 50 papers in international journals and conference proceedings. 